\title{
DETERMINATION OF A VIBRATING BOWL FEEDER DYNAMIC MODEL AND MECHANICAL PARAMETERS
}

\author{
Martin STURM*, Lubomir PESIK ${ }^{*}$ \\ ${ }^{*}$ Faculty of Mechanical Engineering, University of Applied Sciences Zittau/Görlitz, Theodor-Körner-Alleé 16, Zittau, Germany \\ ${ }^{* *}$ Faculty of Mechanical Engineering, Technical University of Liberec, Studentská 2, 46117 Liberec, Czech Republic \\ m.sturm@hszg.de, lubomir.pesik@tul.cz
}

received 13 October 2016, revised 26 September 2017, accepted 27 September 2017

\begin{abstract}
Vibrating conveyors also named bowl feeders are a common equipment for conveying goods into production systems. These systems are used for the supplying of a certain number of goods to an individual designed interface and simultaneously arranging a correct orientation of the goods conveyed by the same time. This type of conveyor is used in various industries, such as for example automotive industry, electronic industry and medical industry. The target of this article is to determine a dynamic model and mechanical parameters by means of testing, and a numerical simulation of a ready-to-operate conveyor under standard working conditions.
\end{abstract}

Key words: Vibrating Conveyor, Bowl Feeder, Resonance Frequencies, Dynamic Model, Mechanical Parameters

\section{INTRODUCTION}

The bowl feeder that was used for determination of the dynamic model and mechanical parameters for future testing and numerical simulations was originally built to forward parts for the electronic industry and is shown in Fig. 1.

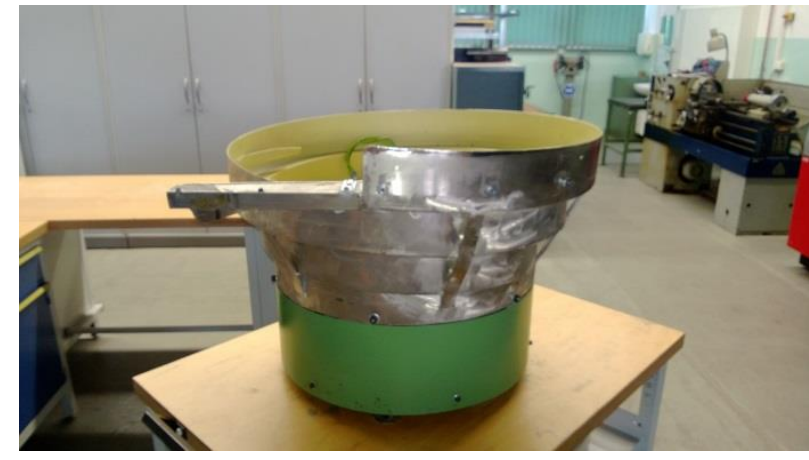

Fig. 1 Bowl feeder for testing

The technical specification of the bowl feeder was as follows in Tab. 1 and in Fig. 2:

Tab. 1. Parameters of the bowl feeder

\begin{tabular}{|c|c|c|c|}
\hline $\mathbf{A}$ & $\mathbf{B}$ & $\mathbf{C}$ & $\mathbf{D}$ \\
\hline $700 \mathrm{~mm}$ & $450 \mathrm{~mm}$ & $400 \mathrm{~mm}$ & $300 \mathrm{~mm}$ \\
\hline $\mathbf{F}$ & $\mathbf{G}$ & $\mathbf{H}$ & $\mathbf{K}$ \\
\hline $535 \mathrm{~mm}$ & $485 \mathrm{~mm}$ & $190 \mathrm{~mm}$ & $48 \mathrm{~mm}$ \\
\hline $\mathbf{L}$ & Total mass & Capacity & Moving mass \\
\hline $130 \mathrm{~mm}$ & $123 \mathrm{~kg}$ & $12 \mathrm{~kg}$ & $33 \mathrm{~kg}$ \\
\hline
\end{tabular}

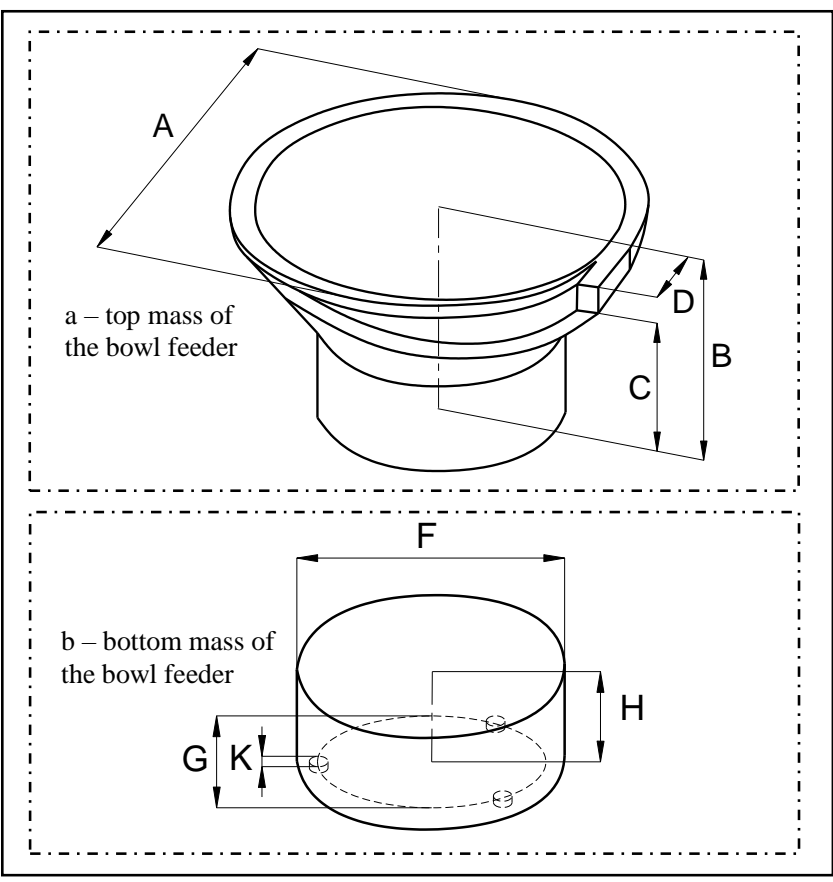

Fig. 2. Parameters of the bowl feeder (http://www.sortimat.com/de/ mediadb/Zufuhr/Downloads/sortimat_ZFTdt_techdata_WF.pdf)

The spring material of the bowl feeder is "Durostone", similar to the present available quality UPM S16 fibre-reinforced plastic. Each of the three springs (flat spring packages) is installed under $15^{\circ}$ to a vertical boundary line. The excitation device is composed by three electromagnetic vibration exciters. Each of them is placed close to the spring (Fig. 3). 


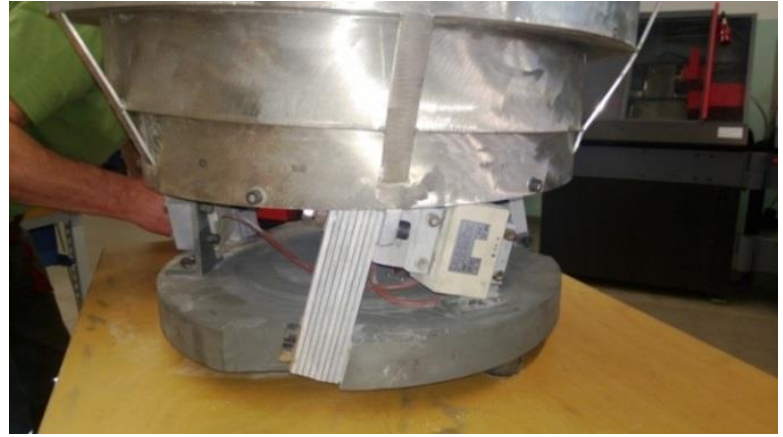

Fig. 3. Springs package with excitation device

\section{DETERMINATION OF THE BOWL FEEDER DYNAMIC MODEL}

The assembly of the bowl feeder includes two principal parts, a conveying element with a conical form and a supporting cylindrical element (Griemert and Römisch, 2015; Sebulke 2017). The first was indexed by character $\mathrm{N}$ and the other by character $\mathrm{S}$. Both parts are connected by springs and they are uniformly distributed around their rotational axes $y_{N}$ and $y_{S}$. In our case, there were used three flat springs packages. Each package was declined by angle $\beta_{N S}$ to the axis plane, it had its stiffness and the total values of all springs packages were defined as $k_{N S}$. The total damping coefficient was described by value $b_{N S}$ (Lanets etal., 2016).

The conveying element was defined by its mass $m_{N}$ and by its moment of inertia $J_{N y}$ around rotational axis $y$. The supporting element had mass $m_{S}$, the moment of inertia $J_{S y}$, and it was placed on rubber springs with total stiffness $k_{S R x}$ and $k_{S R y}$, and with total damping coefficients $b_{S R x}$ and $b_{S R y}$. The whole system was fixed with a rigid connection to the frame (Nendel, 2008; Risch, 2008; Decker, 2014).

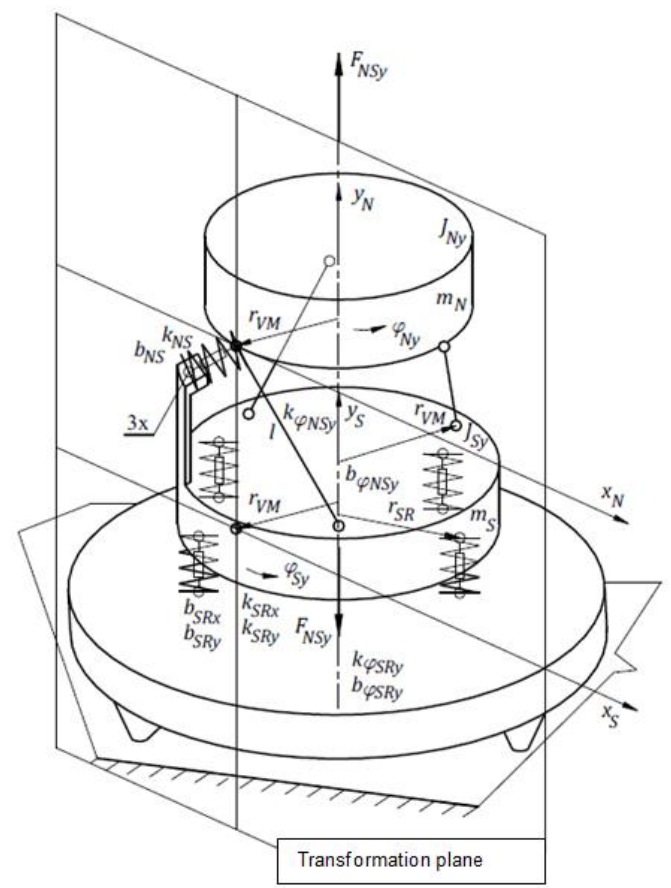

Fig. 5. Mechanical model of the bowl feeder with transformation plane (own source)
In the dynamic model of the system, each flat springs package is replaced by a rod and a spring with a perpendicular effect. All three rods create a joint mechanism between two parts of the bowl feeder (Harris, 2005; Buja, 2007; Mata 2016).

The movement of the bowl feeder system can be described with help of four coordinates, two translations $y_{N}, y_{S}$ and two rotations $\varphi_{N y}, \varphi_{S y}$. The relative movements of both parts are defined:

$y_{N S}=y_{N}-y_{S}$

and

$\varphi_{N S y}=\varphi_{N y}-\varphi_{S y}$.

In these intentions four differential equations describing the movement of the bowl feeder system can be written:

$$
\begin{aligned}
& m_{N} \ddot{y}_{N}+F_{b N S y}+F_{k N S y}+F_{V M} \cos \beta_{N S}=F_{N S y}, \\
& J_{N y} \ddot{\varphi}_{N y}+M_{b N S y}+M_{k N S y}-r_{V M} F_{V M} \sin \beta_{N S}=0, \\
& m_{S} \ddot{y}_{S}+b_{S R y} \dot{y}_{S}-F_{b N S y}+k_{S R y} y_{S}-F_{k N S y}- \\
& -F_{V M} \cos \beta_{N S}=-F_{N S y}
\end{aligned}
$$

and

$J_{S y} \ddot{\varphi}_{S y}+b_{\varphi S R y} \dot{\varphi}_{S y}-M_{b N S y}+k_{\varphi S R y} \varphi_{S y}-M_{k N S y}+$
$+r_{V M} F_{V M} \sin \beta_{N S}=0$,

where force $F_{V M}$ is a connection force between the conveying part and the supporting part which is transferred by the joint mechanism of both parts. Force $F_{N S y}$ is the exciting force between the conveying part and supporting part.

For an example, the following parameters for the characters have been chosen:

$$
\begin{array}{ll}
m_{N}=90 \mathrm{Kg} & F_{N S}=2310 \mathrm{~N} \\
m_{S}=33 \mathrm{Kg} & J_{N}=3,9 \mathrm{Kgm}^{2} \\
\beta=15^{\circ} & J_{S}=1,1 \mathrm{Kgm}^{2} \\
k_{N S}=5698007 \frac{\mathrm{N}}{\mathrm{m}} & b_{N S}=880 \frac{\mathrm{Kg}}{\mathrm{s}}
\end{array}
$$

The other dynamic forces are calculated as follows:

$$
\begin{aligned}
& F_{b N S y}=b_{N S} \dot{y}_{N S}, \\
& F_{k N S y}=k_{N S} y_{N S}, \\
& M_{b N S y}=F_{b N S x} r_{V M}=b_{N S} r_{V M}^{2} \dot{\varphi}_{N S y},
\end{aligned}
$$

and

$M_{k N S y}=F_{k N S x} r_{V M}=k_{N S} r_{V M}^{2} \varphi_{N S y}$,

where:

$F_{b N S x}=b_{N S} \dot{x}_{N S}$,

and

$F_{k N S x}=k_{N S} x_{N S}$.

Torsional damping coefficient $b_{\varphi S R y}$ and torsional stiffness $k_{\varphi S R y}$ are:

$b_{\varphi S R y}=b_{S R x} r_{S R}^{2}$

and 
$k_{\varphi S R y}=k_{S R x} r_{S R}^{2}$.

The relation between relative coordinates $y_{N S}$ and $\varphi_{N S y}$ is done by joint mechanism:

$y_{N S}=\varphi_{N S y} r_{V M} \tan \beta_{N S}$.

In order to make a simplification of the calculation of equations (3) to (6), it is possible to write the balance of forces in the transformation plane (Fig. 6) as follows (Pešík, 2012):

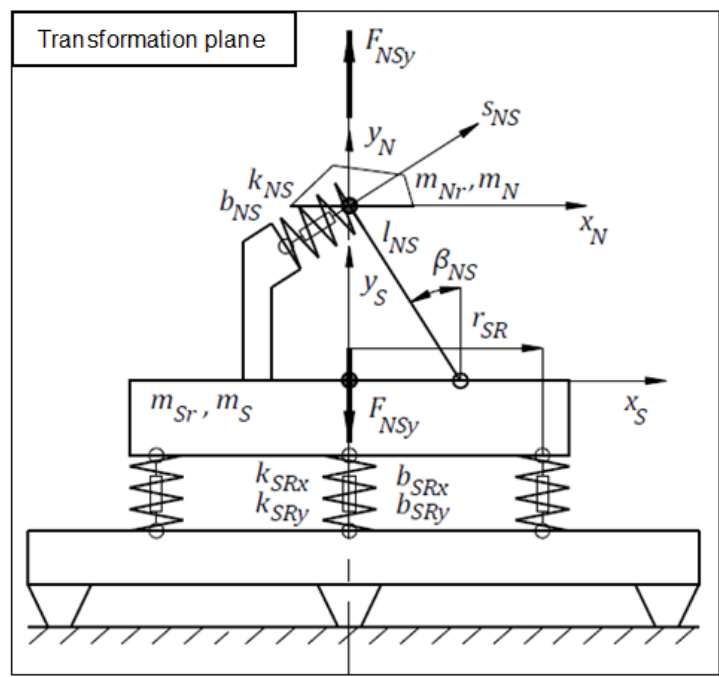

Fig. 6. Dynamic model in the transformation plane (own source)

$x_{N r}=r_{V M} \varphi_{N y}$,

$x_{S r}=r_{V M} \varphi_{S y}$

$x_{N S r}=r_{V M} \varphi_{N S y}$,

$y_{N r}=y_{N}$,

$y_{S r}=y_{S}$,

$y_{N S r}=y_{N S}$

$J_{N y}=m_{N r} r_{V M}^{2}$.

$J_{S y}=m_{S r} r_{V M}^{2}$.

By introduction of coordinate $s_{N S r}$ the number of unknown functions decreases to three:

$x_{N S r}=s_{N S r} \cos \beta_{N S}$

and

$y_{N S r}=s_{N S r} \sin \beta_{N S}$

After the adjustment of the equations (3) to (6), it is possible to write in the matrix form (Decker, 2014):

$\bar{M} \ddot{\bar{q}}+\bar{B} \dot{\bar{q}}+\bar{K} \bar{q}=\bar{Q}$.

where are mass matrix:

$\bar{M}=\left|\begin{array}{ccc}m_{N} \sin ^{2} \beta_{N S}+m_{N r} \cos ^{2} \beta_{N S} & m_{N r} \cos \beta_{N S} & m_{N} \sin \beta_{N S} \\ m_{N r} \cos \beta_{N S} & m_{S r}+m_{N r} & 0 \\ m_{N} \sin \beta_{N S} & 0 & m_{S}+m_{N}\end{array}\right|$ damping matrix:

$\bar{B}=\left|\begin{array}{ccc}b_{N S} & 0 & 0 \\ 0 & b_{S R r} \frac{r_{S R}^{2}}{r_{V M}^{2}} & 0 \\ 0 & 0 & b_{S R y}\end{array}\right|$,

stiffness matrix:

$\bar{K}=\left|\begin{array}{ccc}k_{N S} & 0 & 0 \\ 0 & k_{S R r} \frac{r_{S R}^{2}}{r_{V M}^{2}} & 0 \\ 0 & 0 & k_{S R y}\end{array}\right|$,

coordinates vector:

$\bar{q}=\left|\begin{array}{c}S_{N S r} \\ x_{S R} \\ y_{S R}\end{array}\right|$

and exciting vector:

$\bar{Q}=\left|\begin{array}{c}F_{N S y} \sin \beta_{N S} \\ 0 \\ 0\end{array}\right|$.

From the matrix equation (26) it is to calculate time vector function $\overline{\mathrm{q}}$. The parameters of the bowl feeder system are either known or can be measured. The mass parameters of the conveying and supporting parts are defined in the production documentation. The stiffness and damping parameters are a bit difficult to measure and some differences can be expected between the parameters measured in a static or a dynamic way.

\section{EXPERIMENTAL DETERMINATION OF DYNAMIC PARAMETERS}

Many dynamic parameters can be taken from the technical documentation of the bowl feeder (Tab. 1), the others are calculated or very often measured during the correct excited oscillation.

The primary task of the bowl feeder is the generation of micro throws, and by doing this, the conveyance of goods. Therefore the excitation of bowl feeder free oscillating was realized by a rubber coated hammer in the tangential direction and the oscillation was observed in the vertical direction. For this test, the sensor was mounted vertically under the spiral path of the bowl.

The base for the experimental determination of the dynamic parameters is the measurement of free oscillation kinematic values. The results of this step are time and frequency functions of acceleration (Fig. 7 and Fig. 8).

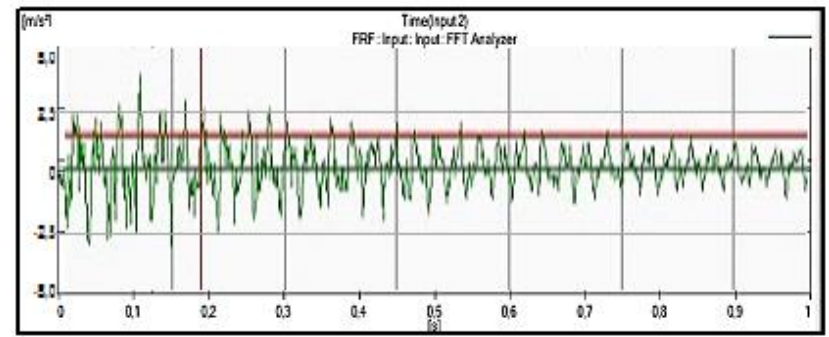

Fig. 7. Time function of acceleration in vertical direction by free oscillation (Machine laboratory, HSZG) 


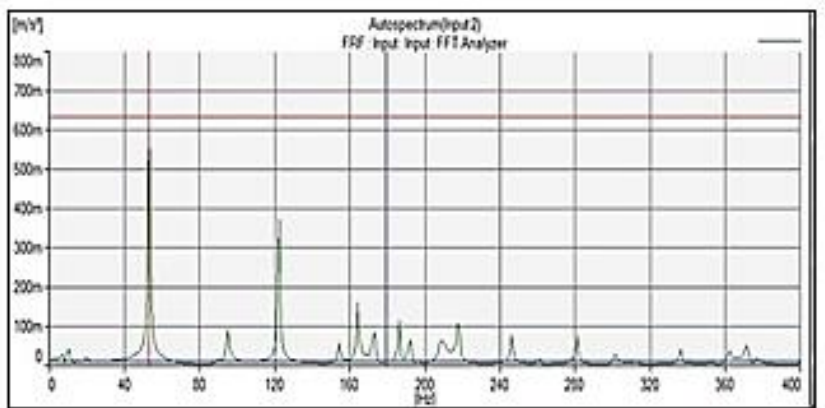

Fig. 8. Frequency function of acceleration in vertical direction by free oscillation (Machine laboratory, HSZG)

As mayor natural frequency, $50 \mathrm{~Hz}$ is identified in Fig. 8. The excitation frequency of the operating bowl feeder is $50 \mathrm{~Hz}$ as well. That shows, the bowl feeder is operation on resonance.

The data supplying necessary information on amplitudes decrease and frequencies. From the time function of acceleration it is possible to find the damping parameters of the bowl feeder system, and the frequency function gives information about its natural frequencies.

In the followed case of the bowl feeder, there is to determine relative weak damping and three principal natural frequencies which correspond to three coordinates of vector function $\overline{\mathrm{q}}$.

The identification of the dynamic parameters is simpler by a reduction of the system, for example - the system with only one degree of freedom. There is considered the stiffness of the rubber springs of the infinitive value. In reality, the supporting part is fixed to the frame and then the equation (26) in case of the free oscillation is reduced to form:

$\left(m_{N} \sin ^{2} \beta_{N S}+\frac{J_{N y}}{r_{V M}^{2}} \cdot \cos ^{2} \beta_{N S}\right) \ddot{s}_{N S}+b_{N S} \dot{S}_{N S}+$ $+k_{N S} s_{N S}=0$.

From the equation (32), values of damping coefficient $b_{\mathrm{NS}}$ and stiffness $k_{N S}$ are determined with help of comparing measured and calculated time function $\ddot{s}_{N S}$ (Lanets et al., 2016).

The simplified system is excited and brought in free oscillating. Measured acceleration $\ddot{s}_{N S}$ of coordinate $s_{N S}$ is shown in Fig. 9 as the time function.

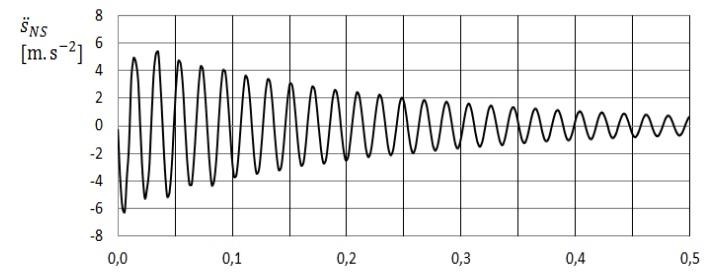

$t[\mathrm{~s}]$

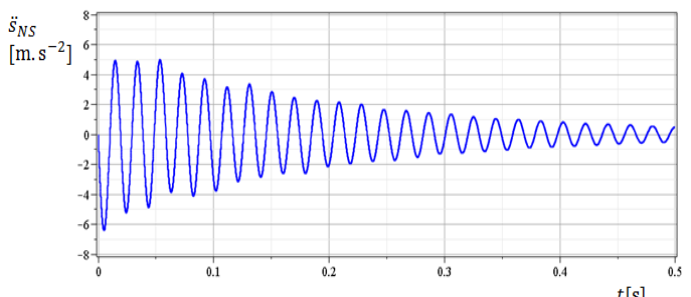

$t[\mathrm{~s}]$

Fig. 9. Time function of acceleration measured (black function) and calculated (blue function) on the simplified system (authors result)
The values of parameters $b_{N S}$ and $k_{N S}$ are chosen so that the measured and calculated time function may show the same or similar curve.

After obtaining of the parameters $b_{N S}$ and $k_{N S}$, the system of the bowl feeder can be returned in the more general configuration as is illustrated in the Fig. 6 and the method is repeated for the parameters $b_{S R x}, b_{S R y}, k_{S R x}$ and $k_{S R y}$.

\section{SUMMARY}

The paper deals with the determination of dynamic parameters of a bowl feeder conducted with a comparison method of simulation and testing results.

The behaviour of the bowl feeder dynamic system is simulated by free oscillation. The results of this simulation are kinematic functions of the bowl feeder oscillation, above all the acceleration time function with data about natural frequencies and the damping of the system. It is advantageous to simplify the dynamic system and thus the kinematic functions, as well, to determine its parameters and advance step by step as far as the full system of the bowl feeder. The same time functions are measured by testing of the bowl feeder in the corresponding configuration of the system. By identical behaviour of simulated and measured functions the dynamic parameters are defined in equations of motion.

\section{REFERENCES}

1. Buja H.O. (2007), Practice Handbook Ram- and Vibrationtechnique (in German), Bauwerk Verlag Berlin.

2. Decker K.H. (2014), Machine elements - Function, Design and calculaion (in German), Carl Hanser Verlag GmbH \& Co. KG.

3. Dresig, H., Holzweißig F. (2008), Machine Dynamics 8 (in German), Auflage, Springer Verlag, Berlin

4. Griemert, R., Römisch, P. (2015): Conveying Technology (in German), Springer Fachmedien Wiesbaden.

5. Harris C.M. (2005), Shock and Vibration Handbook, 5th edition, McGraw-Hill, New York.

6. Lanets O.S., Kachmar R. Ya., Borovets V.M. (2016), Jusitification of parameters of the vibratory hopper feeder with an electromagnetic drive, Industrial Process Automation in Engineering Instrumentation, 50, 54-76.

7. Mata A.S. (2016): Fundamentals of Machine Theory and Mechanisms-Kinematic Analysis of Mechanisms, Springer International Publishing.

8. Nendel K. (2008), Two-Dimensional Motion Form of Virbartory Converyors (in German), http://www.vibrationsfoerdertechnik.de/Dow nload/2D Bewegungsformen_WGTL_Tagungsband08.pdf.

9. Pešík M. (2012), Kinematics of Objects in Vibratory Conveors, ACC Journal, 1st edition, Liberec: Technical University of Liberec, http://acc-ern.tul.cz/cs/journal/item/root/acc-journal-xviii-12012.

10. Risch T. (2008), 2D-Vibration Forms of Vibratory Conveors (in German), http://www.vibrationsfoerder-technik.de/Download/2DBewegungsformen_WGTL_Poster08.pdf.

11. Sebulke, J. (2017): Handbook of Mechanical Engineering (in German), Springer Vieweg, Wiesbaden.

12. Sortimat Technology $\mathrm{GmbH} \&$ Co., Conveyor Technique (in German), http://www.sortimat.com/de/mediadb/Zufuhr/Downloads /sortimat_ZFTdt_techdata_WF.pdf (05.02.2017). 